\title{
Stellarators Resist Turbulent Transport on the Electron Larmor Scale
}

\author{
G. G. Plunk, ${ }^{1,{ }^{*}}$ P. Xanthopoulos, ${ }^{1}$ G. M. Weir, ${ }^{1}$ S. A. Bozhenkov, ${ }^{1}$ A. Dinklage, ${ }^{1}$ G. Fuchert, ${ }^{1}$ J. Geiger, ${ }^{1}$ M. Hirsch, ${ }^{1}$ \\ U. Hoefel, ${ }^{1}$ M. Jakubowski, ${ }^{1}$ A. Langenberg, ${ }^{1}$ N. Pablant, ${ }^{2}$ E. Pasch, ${ }^{1}$ T. Stange, ${ }^{1}$ D. Zhang,,${ }^{1}$ and the W7-X Team \\ ${ }^{1}$ Max-Planck-Institut für Plasmaphysik, Wendelsteinstraße 1, 17491 Greifswald, Germany \\ ${ }^{2}$ Princeton Plasma Physics Laboratory, Princeton, New Jersey 08543, USA
}

(Received 30 July 2018; revised manuscript received 7 November 2018; published 25 January 2019)

\begin{abstract}
Electron temperature gradient (ETG)-driven turbulence, despite its ultrafine scale, is thought to drive significant thermal losses in magnetic fusion devices_-but what role does it play in stellarators? The first numerical simulations of ETG turbulence for the Wendelstein 7-X stellarator, together with power balance analysis from its initial experimental operation phase, suggest that the associated transport should be negligible compared to other channels. The effect, we argue, originates essentially from the geometric constraint of multiple field periods, a generic feature of stellarators.
\end{abstract}

DOI: 10.1103/PhysRevLett.122.035002

Nominally, the thermal transport due to turbulence driven by the electron temperature gradient (ETG), arising on the electron Larmor scale (i.e., "electron-scale" or ETG turbulence), should be weaker by a factor of the square root of the ion-to-electron mass ratio, e.g., $\sqrt{m_{i} / m_{e}} \sim 40$ for hydrogen, as compared to turbulence on the ion Larmor scale ("ion-scale turbulence," e.g., ion-temperaturegradient-driven turbulence); this follows from the mixing length estimates of transport caused by linear modes at disparate scales. In actuality, however, electron-scale turbulence can exceed this estimate because of the operation of different "nonlinear saturation" physics, which can lead to the formation of fingerlike turbulent structures, called "streamers," that convect heat away from the center of the plasma [1]. Since this early discovery, it has been confirmed that ETG turbulence can indeed cause a significant fraction of thermal losses under experimentally realistic conditions when ion-scale instabilities are sufficiently suppressed, such as high- $\beta$ scenarios [2] and plasmas with dominant electron heating [3-6]. In such cases, it is necessary to use computationally expensive multiscale simulations [3,7] to properly resolve the interaction between ion and electron scales. A pressing question is whether Wendelstein 7-X (W7-X), the world's largest fusion facility of the stellarator type, is also confronted with the threat of ETG turbulence. In this Letter, we compare the electron heat transport from a series of numerical simulations of electron-scale turbulence, based on the given profiles, against the experimental levels

Published by the American Physical Society under the terms of the Creative Commons Attribution 4.0 International license. Further distribution of this work must maintain attribution to the author(s) and the published article's title, journal citation, and DOI. inferred from power balance analysis (see, e.g., Weir et al. [8]) for a representative discharge of the first W7-X operation phase [9-11]. The radial variation of the latter, we find, is inconsistent with the physics of the ETG mode, and in fact appears inversely related to simulated levels, leading to the conclusion that ETG turbulence cannot account for the measured transport. Next, we address a complementary scenario, in which the ions are thermally coupled to electrons over the entire radius, as in highdensity plasmas (so-called high-performance discharges) that are more relevant for the production of fusion energy. In this scenario too, our theoretical findings suggest that electron-scale turbulence does not contribute significantly to transport, being in this case greatly exceeded by ion transport levels. We proceeded to analyze our numerical results on fundamental grounds, to determine their physical meaning and generality. We arrive at the conclusion that electron-scale turbulence in the W7-X stellarator may be classified by comparison to two limiting cases, characterized by different power laws of the fluctuation spectrum. One limit is the ideal three-dimensional ("slab") regime, exhibiting a known $-7 / 3$ power law [12], and the other limit is a two-dimensional ("toroidal") regime, giving rise to a $-11 / 3$ power law, which we explain theoretically in terms of a two-dimensional forward cascade of electrostatic energy. Interestingly, both these regimes appear also in the context of ion-scale turbulence [13], with the important distinction that, for the case of electron-scale turbulence, zonal flows play no measurable role in determining which regime is accessible. Instead, it is simple linear physics, as argued by Ref. [1] for tokamaks, that regulates the regime, with the transition between the linear mode branches being essentially controlled by magnetic geometry. However, we note that the absence of toroidal symmetry is not an essential factor, as the behavior of electron-scale turbulence in the W7-X stellarator resembles that in tokamaks with 
negative or small positive magnetic shear, e.g., at the edge region of the device $[14,15]$.

Numerical setup.-The simulations were performed employing the massively parallel code GENE [16], which solves the coupled system of nonlinear gyrokinetic equations. According to this well-established theory [17], the fully kinetic description of the magnetized plasma is reduced by one dimension, owing to the fast gyration of particles about the magnetic field lines. We numerically parameterize the five-dimensional space $\left(x, y, z, v_{\|}, \mu\right)[(x$, $y, z)$ are the radial, binormal, and parallel spatial coordinates; $v_{\|}$is the velocity parallel to the magnetic field; and $\mu$ is the magnetic moment] using a computational box with $120 \times 120 \times 120 \times 40 \times 20$ grid points. The spatial domain sizes are $L_{x}=L_{y}=6 \rho_{s}$ and $L_{z}=2 \pi a$, where $\rho_{s}$ is the ion Larmor radius, and $a$ is a reference minor radius. Moreover, the ETG fluctuations are assumed to be electrostatic (with no magnetic field component). For all simulations, only one species is treated kinetically, neglecting collisions, while the other is assumed to satisfy a Boltzmann distribution.

To facilitate the comparison with analytical theory, the gyrokinetic simulations were performed using a "fluxtube" [18] spatial domain. The magnetic equilibria were generated with the VMEC code [19]. For W7-X, we selected the vacuum "standard" configuration (all nonplanar coils carry the same amount of current), and for the tokamak, we consider circular magnetic surfaces, either with a radially increasing safety factor, which provides the "positiveshear" tokamak ( $\hat{s}=0.5$ on the selected magnetic surface) or with a radially decreasing safety factor, providing the "negative-shear" tokamak $(\hat{s}=-0.5)$. Both tokamak configurations have an aspect ratio of 3.5.

Results.-We can compare our simulation results with findings from Program XP20160309.10 of the first experimental campaign (OP1.1) of the W7-X stellarator. As inferred by the temperature profiles, shown in Fig. 1, the electrons in the core region are much hotter than the ions, due to the thermal decoupling of the species that occurs at low density, although the temperatures become roughly equal toward the edge. These conditions are, in principle, sufficient for the excitation of ETG turbulence over the majority of the plasma volume, since the electron temperature gradient exceeds the density gradient (and even the ion temperature gradient) at most radial positions. However, the radial variation of the electron thermal transport, here represented by the effective electron thermal diffusivity $\chi_{e}^{\text {eff }}$ (inferred from the heat flux, corrected for radiative loss and interspecies exchange; see the middle column in Fig. 1), appears inversely related to that inferred from gyrokinetic simulations $\left(\chi_{e}^{\mathrm{gk}}\right)$. On the other hand, $\chi_{e}^{\text {eff }}$ increases with the density gradient, implicating densitygradient-driven microinstabilities (e.g., trapped-particle instabilities) as its cause; we note that a linear analysis of the $r / a=0.6$ radial point shows that the ETG instability
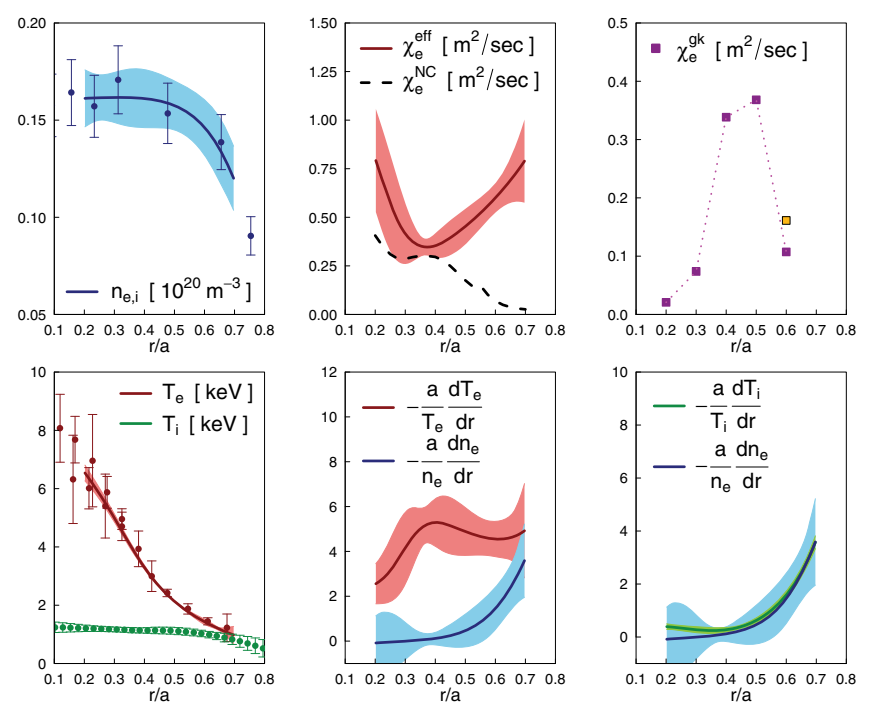

FIG. 1. Density and temperature profiles (left column) with associated gradients (middle and right columns, bottom) and electron thermal diffusivities, for a W7-X OP1.1 discharge, versus normalized radial coordinate; here $n_{i} \approx n_{e}$ is assumed, neglecting impurity density. The hydrogen plasma was ECRHheated by three gyrotrons with a total power of $2 \mathrm{MW}$. Electron heat diffusivities (middle and right columns, top) from power balance analysis $\left(\chi_{e}^{\text {eff }}\right)$ and gyrokinetic simulations $\left(\chi_{e}^{\mathrm{gk}}\right)$ are presented for comparison. The electron density, electron temperature, and ion temperature were measured by Thomsen scattering, ECE radiometry, and an XICS diagnostic, respectively, assuming equal argon and hydrogen ion temperatures [20].

dominates over the trapped electron mode to a degree consistent with dominant ETG turbulence in a tokamak [3]; an additional nonlinear simulation at the same radial location, with the largest value of $a / L_{T e}$ allowed by the error estimate, yields only a minor change in $\chi_{e}^{\mathrm{gk}}$ (yellow point). The initial radial increase of the simulated diffusivity $\chi_{e}^{\mathrm{gk}}$ in Fig. 1 is consistent with the destabilization of the ETG mode with increasing values of the temperature gradient and temperature ratio $\left(T_{i} / T_{e}\right)$, while the subsequent reduction of $\chi_{e}^{\mathrm{gk}}$ in the outer region can be attributed to mode stabilization by the density gradient. It should be noted that the experimental diffusivities contain significant contribution from neoclassical fluxes [20] (also shown in the top middle panel), but the subtraction of this contribution only amplifies the observed trend [21].

We now turn our attention to high-density discharges, where ions and electrons are thermally coupled. Such plasmas are an attractive option for fusion power and will be systematically explored in subsequent W7-X experimental campaigns. In Fig. 2, we compare the ion thermal flux $Q_{i}$ from an ion-scale turbulence simulation in the W7-X stellarator to the electron heat flux $Q_{e}$ from an electron-scale turbulence simulation, setting the electron and ion temperature gradients to the same value. The striking observation here is that the ion thermal flux 


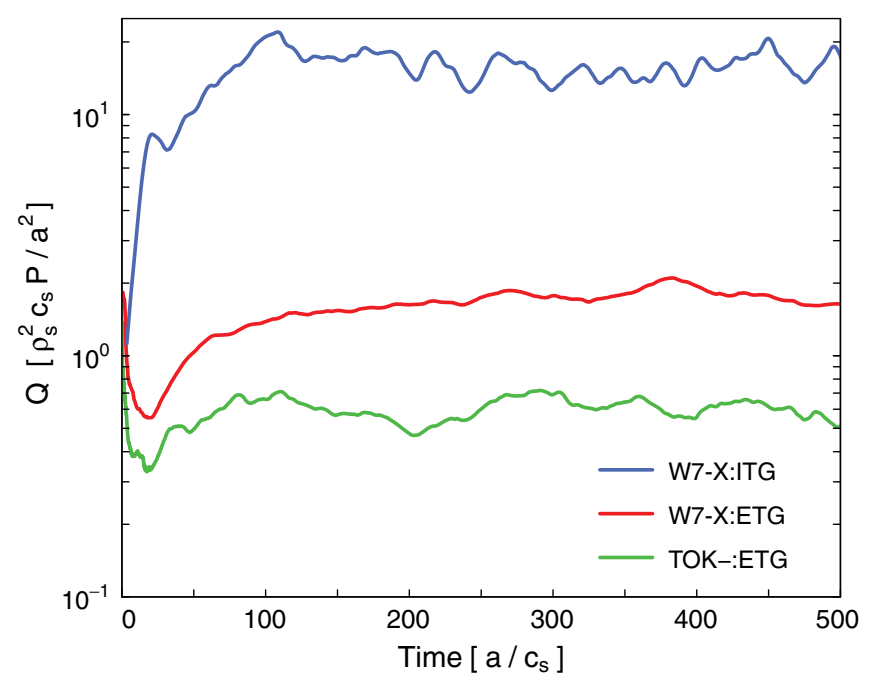

FIG. 2. Comparison of heat fluxes from GENE simulations; $\rho_{s}$ is the ion gyroradius, $c_{s}$ the sound speed, $P$ the ion pressure, and $a$ the minor radius. The heat flux caused by electron-scale turbulence for the W7-X stellarator (W7-X:ETG) and the negative-shear tokamak (TOK - :ETG) are compared to the heat flux caused by equally driven ion-scale turbulence (W7-X: ITG), i.e., $a / L_{T_{i, e}}=3$.

exceeds the electron thermal flux by about 1 order of magnitude. Although the exact value of the ratio of fluxes is dependent on parameters, our simulation results generally imply that the electron contribution should only be a small part of the total thermal flux when the electron and ion profiles are strongly coupled. To gain insight into the significance of the above findings, let us compare the W7-X results with simulations of a tokamak core configuration. It is well known that such simulations, using the adiabatic ion approximation, typically do not reach saturation $[7,22]$ and can produce electron flux levels exceeding the ion flux, even with the inclusion of kinetic ions [16]. The tremendous enhancement of electron fluxes means that electron-scale turbulence must be considered as a key contributor to heat transport, at least in the central part of a tokamak plasma. This notion has spurred the tokamak community to turn to "multiscale" numerical simulations, spanning both electron and ion scales, costing several million CPU hours each [23]. The insignificant fraction of heat flux carried by electron-scale turbulence in the stellarator, on the other hand, as suggested by our findings, implies that much more affordable single-scale simulations might suffice. A significant fact, evidenced by Fig. 2, is that the negative-shear tokamak, like W7-X, also exhibits a low level of heat flux, showing that a 3D magnetic field is not the only possible suppressive mechanism. The suppression caused by magnetic shear has been explained in terms of the different nonlinear saturation mechanisms controlling the amplitude of the two branches of the linear mode [1]. As demonstrated in Fig. 3, the negative-shear tokamak, similar to the stellarator, exhibits oscillatory linear mode

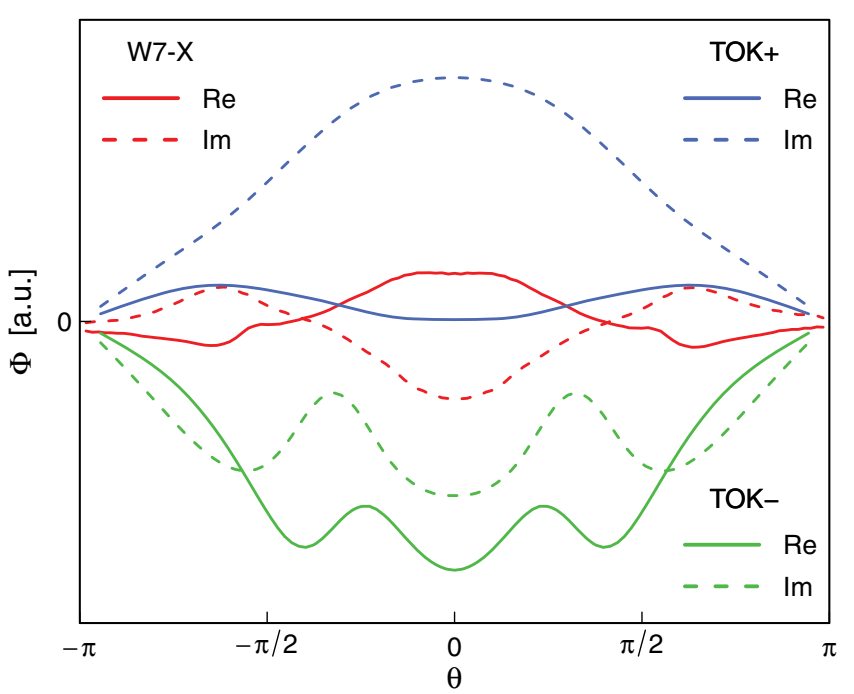

FIG. 3. Mode variation along the magnetic field line for linear modes in the positive-shear tokamak (TOK + ), the negative-shear tokamak (TOK-), and the stellarator (W7-X). For each case, the wave number chosen corresponds to the peak of the nonlinear heat flux spectrum.

structure associated with the slab branch, which saturates by an instability driven by the gradient in parallel electron flow [24]. In the positive-shear tokamak, on the other hand, a strongly toroidal linear mode is excited, subject to a much weaker, two-dimensional instability, causing it to grow to much higher amplitudes. Turning to the nonlinear picture, the toroidal mode excited in the tokamak gives rise to radially elongated streamers, as shown in Fig. 4. Such structures, however, tend to break up in the negative-shear tokamak, and also in the stellarator. Note that the residual, moderately anisotropic structures that survive in W7-X are reminiscent of observations in prior simulations of ETG in the W7-AS stellarator [25].

Although the qualitative features of linear eigenfunctions (see Fig. 3) and density fluctuations (see Fig. 4) are physically intuitive signatures of the turbulence regime, we find that the fluctuation spectrum $E\left(k_{y}\right)=\sum_{k_{x}}|\hat{\phi}|^{2} / 2$ can be used as a quantitative measure. In the collisionless limit, two theoretical extremes exist: (i) the fully 3D (slab) limit $E \sim k_{y}^{-7 / 3}$, which has been observed in ion-scale turbulence [12] (note the collisional corrections found by Ref. [26]), and (ii) the 2D (toroidal) limit $E \sim k_{y}^{-11 / 3}$, for which we now outline a new cascade model based on the two-dimensional fluid limit of gyrokinetics. Neglecting linear terms, the following equation may be obtained in the limit $k_{\perp}^{2} \rho_{e}^{2} \ll 1$ (the detailed derivation will be published later):

$\partial_{t} \varphi+\hat{\mathbf{z}} \times \boldsymbol{\nabla} \varphi \cdot \boldsymbol{\nabla}\left(-\nabla^{2} \varphi-\nabla^{2} \chi\right)+\hat{\mathbf{z}} \times \boldsymbol{\nabla} \chi \cdot \nabla\left(-\nabla^{2} \varphi\right)=0$, 


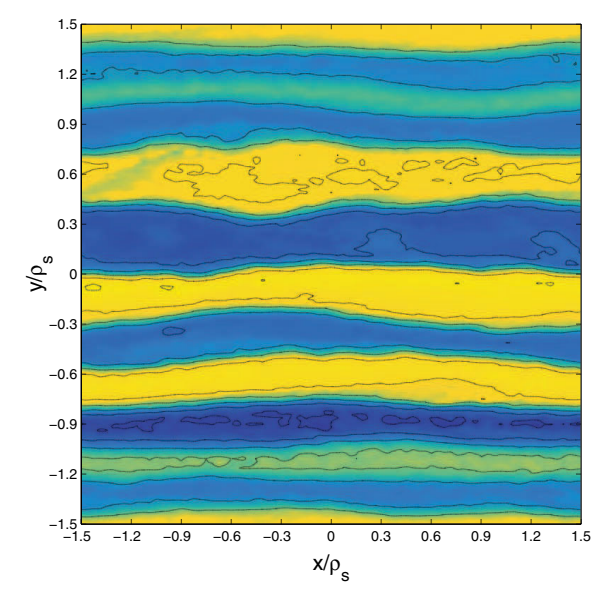

(a) TOK+ configuration.

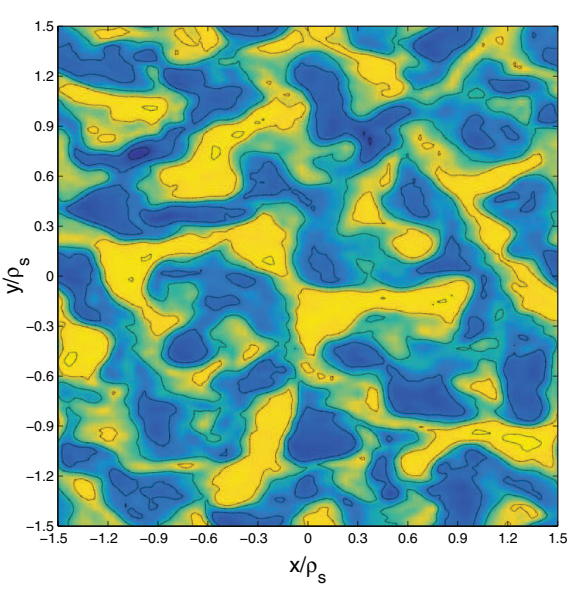

(b) TOK- configuration.

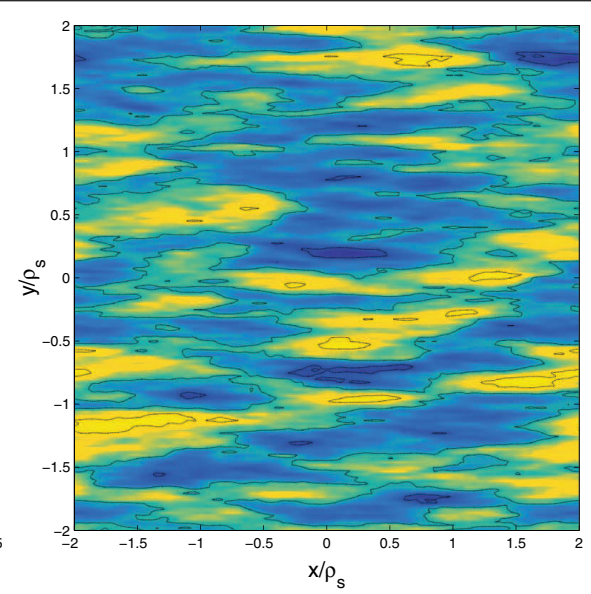

(c) W7-X stellarator.

FIG. 4. Density fluctuations from GENE simulations of electron-scale turbulence plotted in the plane perpendicular to the magnetic field. (a) The positive-shear tokamak (TOK+), (b) the negative-shear tokamak (TOK-), and (c) the stellarator Wendelstein-7X are compared. Here, $x$ denotes the radial coordinate and $y$ the binormal coordinate.

where $\chi$ is the perturbed normalized perpendicular electron pressure, and $\varphi$ is the normalized electrostatic potential. The electrostatic energy $E=\int d x d y \varphi^{2} / 2$ is conserved under nonlinear interactions for this system, but, unlike the closely related Hasegawa-Mima equation (valid only for cold ions), this energy can cascade in the forward sense (to smaller scales), due to the absence of an additional quadratic invariant analogous to enstrophy. From this point, it is straightforward to demonstrate that such a cascade leads to the spectrum $E\left(k_{y}\right) \sim k_{y}^{-11 / 3}$. This power law seems to be a good match for the positiveshear tokamak, as shown in Fig. 5, while both the negative-shear tokamak and the W7-X stellarator appear close to the pure slab limit.

Geometric control of mode branch.- Since the nonlinear regime is fundamentally linked to linear physics, we examine the linear theory of the ETG mode in order to determine a condition regulating the transition between the two branches of the mode. This condition, we find, is of purely geometric nature, i.e., independent of plasma conditions, like the temperature gradient. We consider an equation for $\hat{\phi}$, the amplitude of the ETG mode, formally valid in the nonresonant limit at a low trapped particle fraction. In a normalized form, this equation reads [see, e.g., Eq. (15) of Ref. [27] ]

$\left[\hat{\omega}^{3}-b_{*} g(\vartheta) \hat{\omega}^{2}+f(\vartheta) \hat{\omega}+\frac{R_{\mathrm{c}}}{4 b_{*} L_{\|}} \frac{d^{2}}{d \vartheta^{2}}\right] \hat{\phi}(\vartheta)=0$.

Note that we neglect the density gradient, which stabilizes both branches. In equation (2), the frequency is normalized by the nominal growth rate of the toroidal ETG mode, $\hat{\omega}=\omega \sqrt{R_{\mathrm{c}} L_{T e}} /\left(v_{\mathrm{th}, e} k_{y} \rho_{e}\right)$ (where $k_{y}$ is the binormal wave number; $\rho_{e}$ is the electron Larmor radius; $v_{\text {th.e }}$ is the electron thermal velocity; $R_{\mathrm{c}}$ is the local radius of magnetic curvature, here assumed comparable to the device major radius; and $L_{T e}$ is the electron temperature gradient length), and therefore $\hat{\omega} \sim 1$ when the mode is toroidal. The equation is differential in the angular coordinate $\vartheta=\pi l / L_{\|}$, i.e., the arc length along the field line $l$ divided by the connection length $L_{\|}$. This length is defined such that the dimensionless coefficients $f$ and $g$ vary on the scale $\Delta \vartheta \sim \pi$. Therefore, to be identified as toroidal, a mode must be localized to a region of this scale, implying $d / d \vartheta \gtrsim 1$.

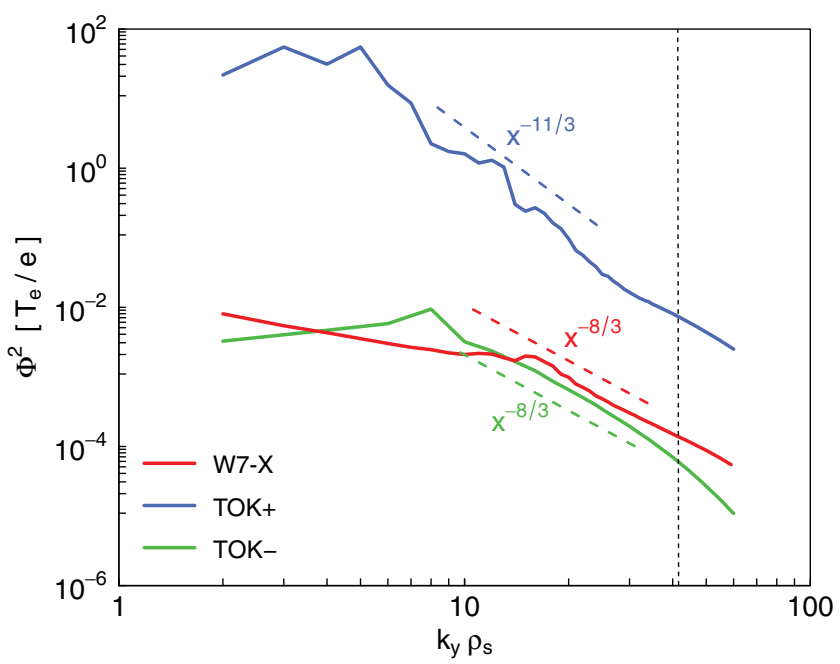

FIG. 5. Fluctuation spectra for electron-scale turbulence compared against power laws. The negative-shear tokamak (TOK-) and W7-X configurations are compared with $k_{y}^{-8 / 3}$, which is slightly steeper than the slab limit $k_{y}^{-7 / 3}$, while the positive-shear tokamak $(\mathrm{TOK}+)$ matches the theoretical law $k_{y}^{-11 / 3}$, corresponding to two-dimensional (toroidal) turbulence. Note that these power laws apply for wave numbers larger than the injection scale and less than $k_{y} \rho_{e}=1$ (vertical line). 
The function $g(\vartheta)$ captures the variation along the field line of the finite-Larmor-radius term, while $f(\vartheta)$ plays an analogous role for the toroidal drive; they are defined such that $f(0)=g(0)=1$. The remaining dimensionless constants are $L_{\|} / R_{\mathrm{c}}$ and $b_{*}=k_{y}^{2} \rho_{e}^{2} \sqrt{R_{\mathrm{c}} / L_{T e}}$. Since the finiteLarmor-radius term is stabilizing, we must have $b_{*}<1$. Furthermore, we observe that the differential term must also be small for the mode to be toroidal, so, since $d / d \vartheta \gtrsim 1$, we find $L_{\|} / R_{\mathrm{c}}>1 / b_{*}$, and we therefore conclude that $L_{\|} / R_{\mathrm{c}}>1$ is required for the existence of a toroidal mode. In any toroidal configuration, properties of the magnetic geometry, such as negative magnetic shear, can cause the connection length to be smaller than the usual estimate $L_{\|} \sim q R_{\mathrm{c}}$ (where $q$ is the safety factor), allowing access to the slab regime, $L_{\|} / R_{\mathrm{c}}<1$. Stellarators benefit from this effect by design, as they have multiple field periods, a feature which limits the connection length, since geometric quantities like curvature and local magnetic shear vary strongly from one field period to the next [28]. We can estimate a bound on the connection length in a stellarator as $L_{\|} \lesssim R_{\mathrm{c}} / N$, where $N$ is the field period number (e.g., $N=5$ for W7-X). This implies $L_{\|} / R_{\mathrm{c}} \sim$ $1 / N<1$. It is due to these reasons that the linear modes in a stellarator exhibit more of a slablike character than tokamaks, as has been observed in linear simulations of W7-X, by measuring the relative energetic importances of the parallel and perpendicular resonances [29].

Conclusion.- The first gyrokinetic simulations of electron-scale turbulence in the W7-X fusion device, compared with a power balance analysis for a discharge from the initial phase of the experiment, suggest that ETG turbulence, under conditions where it is expected to be dominant, cannot account for the observed electron heat transport. Because of the mild, slablike nature of such turbulence in a stellarator, we expect electron-scale transport to be insignificant for reactor-relevant W7-X plasma parameters. Considering the stabilization of trapped-particle turbulence in the quasi-isodynamic limit [30,31], as can be approached in W7-X, together with the potential suppression of ionscale turbulence in the outer plasma by the neoclassical electric field [32], our results further bolster the expectations to find high-performance operational regimes in stellarators.

The authors thank F. Jenko, T. Görler, F. Warmer, M. Beurskens, D. Hatch, D. Told, M. Landreman, P. Helander, and Y. Turkin for physics discussions and technical support. The GENE simulations were performed on the RZG (Germany) and Marconi (Italy) supercomputers. This work has been carried out within the framework of the EUROfusion Consortium and has received funding from the Euratom research and training programs under Grant Agreement No. 633053. The views and opinions expressed herein do not necessarily reflect those of the European Commission. *gplunk@ipp.mpg.de

[1] F. Jenko and W. Dorland, Phys. Rev. Lett. 89, 225001 (2002).

[2] S. Maeyama, Y. Idomura, T.-H. Watanabe, M. Nakata, M. Yagi, N. Miyato, A. Ishizawa, and M. Nunami, Phys. Rev. Lett. 114, 255002 (2015).

[3] T. Görler and F. Jenko, Phys. Rev. Lett. 100, 185002 (2008).

[4] N. Howard, C. Holland, A. White, M. Greenwald, and J. Candy, Nucl. Fusion 56, 014004 (2016).

[5] C. Holland, N. Howard, and B. Grierson, Nucl. Fusion 57, 066043 (2017).

[6] N. Bonanomi, P. Mantica, J. Citrin, T. Goerler, B. Teaca, and J. Contributors, Nucl. Fusion 58, 124003 (2018).

[7] R.E. Waltz, J. Candy, and M. Fahey, Phys. Plasmas 14, 056116 (2007).

[8] G. Weir, B. J. Faber, K. M. Likin, J. N. Talmadge, D. T. Anderson, and F. S. B. Anderson, Phys. Plasmas 22, 056107 (2015).

[9] T. S. Pedersen et al., Nucl. Fusion 55, 126001 (2015).

[10] S. Bozhenkov, F. Effenberg, Y. Feng, J. Geiger, D. A. Hartmann, H. Hölbe, T. S. Pedersen, and R. C. Wolf, in 41st EPS Conference on Plasma Physics, Europhysics Conference Abstracts (ECA) Vol. 38F, edited by S. Ratynskaia, P. Mantica, A. Benuzzi-Mounaix, G. Dilecce, R. Bingham, M. Hirsch, B. Kemnitz, and T. Klinger (European Physical Society, Berlin, 2014).

[11] Dinklage et al., Nat. Phys. 14, 855 (2018).

[12] M. Barnes, F. I. Parra, and A. A. Schekochihin, Phys. Rev. Lett. 107, 115003 (2011).

[13] G. G. Plunk, P. Xanthopoulos, and P. Helander, Phys. Rev. Lett. 118, 105002 (2017).

[14] D. Told, F. Jenko, P. Xanthopoulos, L. D. Horton, E. Wolfrum, and A. U. Team, Phys. Plasmas 15, 102306 (2008).

[15] D. Hatch, D. Told, F. Jenko, H. Doerk, M. Dunne, E. Wolfrum, E. Viezzer, T. A. U. Team, and M. Pueschel, Nucl. Fusion 55, 063028 (2015).

[16] F. Jenko, W. Dorland, M. Kotschenreuther, and B.N. Rogers, Phys. Plasmas 7, 1904 (2000); http://www .genecode.com/.

[17] A. J. Brizard and T. S. Hahm, Rev. Mod. Phys. 79, 421 (2007).

[18] M. A. Beer, S. C. Cowley, and G. W. Hammett, Phys. Plasmas 2, 2687 (1995).

[19] S. P. Hirschman, Phys. Fluids 26, 3553 (1983).

[20] N. A. Pablant et al., Phys. Plasmas 25, 022508 (2018).

[21] Please note that Ref. [20] used the Thomsen scattering diagnostic to measure $T_{e}$, while we have used the ECE diagnostic due to increased spatial resolution. The two diagnostics are consistent within uncertainties.

[22] W. M. Nevins, J. Candy, S. Cowley, T. Dannert, A. Dimits, W. Dorland, C. Estrada-Mila, G. W. Hammett, F. Jenko, M. J. Pueschel, and D. E. Shumaker, Phys. Plasmas 13, 122306 (2006).

[23] T. Görler, F. Jenko, M. J. Pueschel, and H. Lesch, in Gyrokinetic Turbulence Investigations Involving Ion and Electron Scales, edited by S. Wagner, M. Steinmetz, A. Bode, and M. Müller, High Performance Computing in Science and Engineering (Springer, Berlin, Heidelberg, 2009). 
[24] S. C. Cowley, R. M. Kulsrud, and R. Sudan, Phys. Fluids B 3, 2767 (1991).

[25] F. Jenko and A. Kendl, Phys. Plasmas 9, 4103 (2002).

[26] D. R. Hatch, F. Jenko, A. Bañón Navarro, and V. Bratanov, Phys. Rev. Lett. 111, 175001 (2013).

[27] G. G. Plunk, P. Helander, P. Xanthopoulos, and J. W. Connor, Phys. Plasmas 21, 032112 (2014).

[28] P. Helander, C. D. Beidler, T. M. Bird, M. Drevlak, Y. Feng, R. Hatzky, F. Jenko, R. Kleiber, J. H. E. Proll, Y. Turkin, and P. Xanthopoulos, Plasma Phys. Controlled Fusion 54, 124009 (2012).

[29] V. Kornilov, R. Kleiber, R. Hatzky, L. Villard, and G. Jost, Phys. Plasmas 11, 3196 (2004).

[30] J. H. E. Proll, P. Helander, J. W. Connor, and G. G. Plunk, Phys. Rev. Lett. 108, 245002 (2012).

[31] P. Helander, J. Plasma Phys. 83, 715830401 (2017).

[32] J. Riemann, R. Kleiber, and M. Borchardt, Plasma Phys. Controlled Fusion 58, 074001 (2016). 